\title{
Functional Regulator Therapy in Treatment of Skeletal Open-bite
}

\author{
Bülent HAYDAR ${ }^{1}$ and Ayhan ENACAR ${ }^{2}$
}

(Received 13 November 1991 and accepted 3 July 1992)

Key words: Fränkel appliance, open bite, functional regulator

\begin{abstract}
A study was performed on the functional regulator, Fränkel appliance (FR4) in order to test its efficiency in the treatment of patients with skeletal open-bite. Pretreatment and post-treatment cephalometric evaluation was done on 11 patients and 10 untreated patients with skeletal open-bite. The results showed that the FR4 appliance was mainly effective on changes in dentoalveolar structures and produced no significant skeletal changes. The degree of anterior open-bite was decreased significantly in the treatment group in comparison with the controls $(p<0.01)$, due to vertical eruption of upper and lower incisors and retraction of maxillary incisors.
\end{abstract}

\section{Introduction}

Treatment of skeletal anterior open-bite deformity is one of the most difficult challenges for the orthodontist. The main cephalometric characteristics of this malocclusion are a decrease in the ratio of posterior to anterior face hight ${ }^{[1-5]}$, an increase in anterior face height ${ }^{[1-8]}$, due mainly to a rise in lower anterior face height and the mandibular plane angle as a result of backward rotation of the mandible, and a rise in the posterior and anterior maxillary and mandibular dental height ${ }^{[2,6,8,9]}$. Extraorally, affected patients have a narrow alar base and a parted lips posture which is a characteristic feature of their mouth breathing ${ }^{[10]}$.

The etiology of this malocclusion may be multifactorial including heredity, sucking habits, mouth breathing with associated head-posture and some developmental anomalies ${ }^{[11]}$. Various methods of orthodontic treatment have been used with reference to the etiology of open-bite malocclusion ${ }^{[9,10,12-16]}$. Depending on the age of the patient, a functional therapy approach during the mixed dentition period or fixed appliance therapy after establishment of permanent dentition may be used.

Moss et al. ${ }^{[17]}$ states that capsular functional matrices may play an important role in open-bite. Either the form of the oral functional space or its location may be abnormal relative to the nasal and pharyngeal functional spaces. On the basis of this concept, FRÄNKEL AND FRÄNKEL ${ }^{[18]}$ developed a functional approach to

\footnotetext{
Based on a thesis submitted in partial fulfillment of the requirements for the Master of Science degree, Faculty of Dentistry, University of Hacettepe

1 Department of Orthodontics, Faculty of Dentistry, Hacettepe University.

2 Department of Orthodontics, Faculty of Dentistry, Hacettepe University.

To whom all correspondence should be addressed: Dr. Bülent HAYDAR, Başak Sokak, 45/21, Küçükesat 06660, Ankara, TURKEY.
} 
orofacial orthopedics, and introduced the FR4 appliance for the treatment of skeletal open-bite malocclusion. This works by correcting the faulty postural activity of the orofacial musculature and helps to correct the associated skeletal deformity. It has also been claimed that this approach reverses the backward rotational growth pattern of the mandible.

The aim of the present study was to determine the effects of the FR4 appliance on the developing dentofacial skeletal structures in patients with skeletal openbite. Cephalometric comparisons of the treated and control groups were made to evaluate the effects of the appliance.

\section{Materials and Methods}

Eleven patients in the mixed dentition period who had an anterior open-bite were treated with the FR4 appliance at the postgraduate orthodontic clinic of Hacettepe University. In addition, 10 children in the mixed dentition period with the same type of malocclusion were used as a control group. Average ages at the beginning and end of the observation period are shown in Table I. The patients were selected according to the following criteria: 1-Presence of anterior open-bite with a vertical growth pattern. 2-Lack of sucking habits. 3-Patients were in their mixed dentition period.

Table I

Average ages at the beginning of treatment and duration of treatment

\begin{tabular}{|l|c|c|c|c|c|}
\cline { 2 - 6 } \multicolumn{1}{c|}{} & $\mathrm{n}$ & Average age & $\mathrm{SD}$ & $\begin{array}{c}\text { Duration of } \\
\text { treatment }\end{array}$ & $\mathrm{SD}$ \\
\hline Treatment group & 11 & 8.773 & 1.174 & 1.235 & 0.241 \\
Control group & 10 & 8.284 & 1.064 & 1.024 & 0.039 \\
\hline
\end{tabular}

Lateral cephalograms of all patients were taken before and after the observation or treatment period. The FR4 appliance was constructed according to the methods described by Fränkel AND FRÄNKeL ${ }^{[19]}$ and Graber et al. ${ }^{[20]}$ (Fig. 1, a and $b$ ).

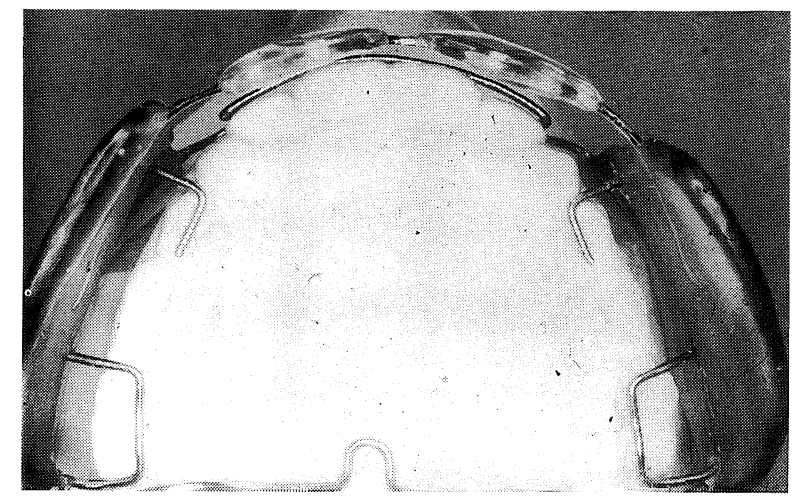

Fig. 1 a The FR 4 appliance on a maxillary model showing the occlusal rests on permanent and deciduous first molars 


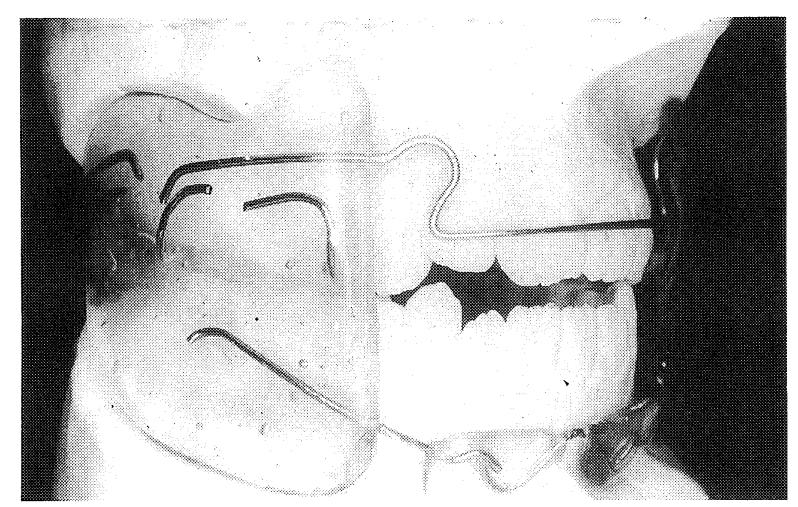

Fig. $1 \mathrm{~b}$ Finished appliance tested on mounted models before applying to the mouth

The patients were instructed to wear the appliance for $3 \mathrm{~h}$ on the first day and add one hour each day for the first ten days. After this period the patients were instructed to wear the appliance full-time (at least $20 \mathrm{~h}$ per day) except during eating or sports activity. They were advised not to wear the appliance during sleeping hours during the first 10 days. The importance of lip seal exercises were explained to the patients, and they, were instructed to keep their lips together as much as possible. The patients were instructed to perform lip seal exercises such as holding a coin between the lips, blowing up a balloon or whistling.

Patients with upper airway problems were told to consult an ear-nose-throat (ENT) specialist, and adequate treatment measures were taken whenever necessary.

The pre- and post-treatment lateral cephalograms were traced, and 21 anatomic landmarks were used; 35 parameters -16 angular and 19 linear-were used to evaluate the effects of the FR4 appliance. Cephalometric evaluation was carried out on maxillary and mandibular skeletal and dental structures and also on vertical changes.

Statistical evaluation was made by Wilcoxon signed-rank test for longitudinal changes and Mann-Whitney U test for comparison of two groups.

\section{Findings}

The data showed that before treatment the FR4 group had more retroclined maxillary incisors than the controls (U1-SN ${ }^{\circ} \mathrm{p}<0.05$, U1-NA $\left.{ }^{\circ} \mathrm{p}<0.05\right)$ (Table II). In addition the FR4 group displayed higher U1-PP( $\mathrm{mm})$ and $\mathrm{U} 6-\mathrm{FH}(\mathrm{mm})$ values $(\mathrm{p}<0.05)$ (Table II).

Maxillary skeletal and dental changes (Tables III, IV, V)

The Fränkel appliance appeared to restrain maxillary growth slightly. Maxillay length (Harvold) increased $1.227 \mathrm{~mm}$ in the treatment group $(\mathrm{p}<0.05)$ and $2.250 \mathrm{~mm}$ in the control group $(\mathrm{p}<0.01)$.

Maxillary incisors showed a significant amount of angular retraction (U1-SN ${ }^{\circ}$ : $-3.864^{\circ} \mathrm{p}<0.05$, U1-NA: $-3.091^{\circ} \mathrm{p}<0.05$ ) and bodily retraction (U1-NAmm: -1.136 $\mathrm{p}<0.05)$. Upon comparison of these changes with the control group, only the decrease in the U1-NAmm measurement was found to be significant $(p<0.05)$. 
Table II

Pretreatment comparison of FR4 and control groups

\begin{tabular}{|c|c|c|c|c|}
\hline Parameter & $\begin{array}{l}\text { Experimental } \\
(\bar{x} \pm S D)\end{array}$ & $\begin{array}{l}\text { Control } \\
(\bar{x} \pm S D)\end{array}$ & $\mathrm{U}$ & $\mathrm{p}$ value \\
\hline \multicolumn{5}{|l|}{ Maxillary skeletal and dental } \\
\hline SNA $\left(^{\circ}\right)$ & $74.864 \pm 12.339$ & $78.450 \pm 7.065$ & 49 & 0.671 \\
\hline N. perpendicular-A (mm) & $-0.136 \pm 2.226$ & $-1.800 \pm 3.450$ & 69 & 0.339 \\
\hline Maxillary length(mm) (Cd-A) & $78.500 \pm 2.377$ & $75.150 \pm 4.710$ & 82 & 0.061 \\
\hline Convexity $(\mathrm{mm})$ & $5.409 \pm 1.985$ & $3.700 \pm 1.874$ & 82 & 0.053 \\
\hline $\mathrm{SN}-\mathrm{PP}\left({ }^{\circ}\right)$ & $10.045 \pm 3.275$ & $8.900 \pm 4.683$ & 66 & 0.459 \\
\hline PP-MP $\left({ }^{\circ}\right)$ & $34.000 \pm 4.764$ & $32.600 \pm 4.142$ & 68 & 0.377 \\
\hline Occ-PP $\left({ }^{\circ}\right)$ & $20.500 \pm 4.806$ & $17.450 \pm 3.715$ & 79 & 0.097 \\
\hline Occ-MP $\left(^{\circ}\right)$ & $13.500 \pm 2.898$ & $15.150 \pm 2.161$ & 33 & 0.119 \\
\hline U1-SN $\left({ }^{\circ}\right)$ & $98.591+6.587$ & $104.050 \pm 5.718$ & 21 & $0.016^{*}$ \\
\hline U1-NA $\left(^{\circ}\right)$ & $21.045 \pm 3.971$ & $25.900 \pm 6.887$ & 27 & $0.048^{*}$ \\
\hline U1-NA (mm) & $2.727 \pm 1.348$ & $2.850 \pm 1.415$ & 51 & 0.774 \\
\hline U1-FH (mm)(dental height) & $46.818 \pm 3.364$ & $43.150 \pm 4.177$ & 81 & 0.071 \\
\hline U1-PP (mm) (dental height) & $26.818 \pm 1.793$ & $24.100 \pm 3.035$ & 86 & $0.031^{*}$ \\
\hline U6-FH (mm) (dental height) & $42.045 \pm 2.945$ & $34.450 \pm 2.477$ & 90 & $0.014^{*}$ \\
\hline U6-PP (mm) (dental height) & $20.955 \pm 2.173$ & $19.400 \pm 1.524$ & 79 & 0.089 \\
\hline \multicolumn{5}{|c|}{ Mandibular skeletal and dental } \\
\hline $\operatorname{SNB}\left({ }^{\circ}\right)$ & $71.909 \pm 4.493$ & $74.350 \pm 6.223$ & 39 & 0.259 \\
\hline Facial angle $\left({ }^{\circ}\right)$ & $84.045 \pm 3.602$ & $83.700 \pm 2.965$ & 55 & 0.972 \\
\hline N. perpendicular-Pog (mm) & $11.139 \pm 5.595$ & $11.000 \pm 5.533$ & 58 & 0.832 \\
\hline Mandibular lenth(mm)(Cd-Gn) & $102.364 \pm 4.348$ & $98.750 \pm 5.633$ & 80 & 0.078 \\
\hline Ramus height (mm) & $50.864 \pm 3.661$ & $48.000 \pm 5.121$ & 74 & 0.180 \\
\hline Gonial angle $\left(^{\circ}\right)$ & $127.273 \pm 4.239$ & $127.400 \pm 4.458$ & 51 & 0.777 \\
\hline L1-MP $\left({ }^{\circ}\right)$ & $91.773 \pm 5.183$ & $92.900 \pm 5.806$ & 50 & 0.698 \\
\hline L1-NB $\left({ }^{\circ}\right)$ & $26.682 \pm 3.723$ & $28.100 \pm 5.705$ & 50 & 0.723 \\
\hline L1-NB (mm) & $4.727 \pm 1.403$ & $5.150 \pm 2.334$ & 53 & 0.887 \\
\hline L1-MP (mm) (dental height) & $38.000 \pm 2.280$ & $36.500 \pm 2.582$ & 81 & 0.070 \\
\hline L6-MP (mm) (dental height) & $31.182 \pm 1.820$ & $29.750 \pm 2.276$ & 77 & 0.118 \\
\hline \multicolumn{5}{|l|}{ Vertical } \\
\hline Facial axis $\left({ }^{\circ}\right)$ & $79.045 \pm 4.156$ & $80.950 \pm 3.166$ & 39 & 0.244 \\
\hline FMA $\left(^{\circ}\right)$ & $31.636 \pm 5.745$ & $32.150 \pm 3.266$ & 52 & 0.804 \\
\hline Facial height(\%)(UFH/LFH) & $0.751 \pm 0.049$ & $0.766 \pm 0.081$ & 53 & 0.858 \\
\hline Lower face height $\left(^{\circ}\right)$ & $49.045 \pm 3.984$ & $48.400 \pm 3.017$ & 61 & 0.696 \\
\hline Posterior face height $(\mathrm{mm})(\mathrm{S}-\mathrm{Go})$ & $68.045 \pm 4.569$ & $66.850 \pm 5.011$ & 64 & 0.549 \\
\hline Anterior face height $(\mathrm{mm})(\mathrm{N}-\mathrm{Me})$ & $115.500 \pm 6.961$ & $111.100 \pm 3.872$ & 76 & 0.147 \\
\hline Jarabak ratio (\%) & $58.900 \pm 4.400$ & $59.900 \pm 4.500$ & 45 & 0.479 \\
\hline Mandibular arc $\left({ }^{\circ}\right)$ & $34.955 \pm 3.236$ & $33.250 \pm 4.316$ & 64 & 0.524 \\
\hline Anterior open-bite (mm) & $2.636 \pm 1.675$ & $3.800 \pm 1.317$ & 80 & 0.072 \\
\hline
\end{tabular}

${ }^{*} \mathrm{p}<0.05$

Although significant extrusion of the upper incisors was found (U1-FHmm: $3.227 \mathrm{~mm} \mathrm{p}<0.01$, U1-PP mm: $1.864 \mathrm{~mm} \mathrm{p}<0.01$ ), these changes were not significant when compared with the control $(p>0.05)$. Similarly, upper 1. molars showed a noticeable increase in vertical height $(p<0.05)$, but this was not found to be significant when compared with the control $(\mathrm{p}>0.05)$.

Mandibular skeletal and dental changes (Tables III, IV, V)

No measurements in these groups were found to be significantly noteworthy when the two groups were compared.

Although significant increases in the mandibular length and ramus height 
Table III

Longitudinal changes in the treatment group

\begin{tabular}{|c|c|c|c|c|}
\hline Parameter & $\begin{array}{l}\text { Pretreatment } \\
(\bar{x} \pm S D)\end{array}$ & $\begin{array}{l}\text { Post-treatment } \\
(\bar{x} \pm S D)\end{array}$ & Difference & $\mathrm{p}$ value \\
\hline \multicolumn{5}{|l|}{ Maxillary skeletal and dental } \\
\hline SNA $\left({ }^{\circ}\right)$ & $74.864 \pm 12.339$ & $77.409 \pm 4.421$ & -0.455 & 0.477 \\
\hline N. perpendicular-A (mm) & $-0.136 \pm 2.226$ & $-0.955 \pm 3.150$ & -0.818 & 0.051 \\
\hline Maxillary length $(\mathrm{mm})(\mathrm{Cd}-\mathrm{A})$ & $78.500 \pm 2.377$ & $79.727 \pm 2.494$ & 1.227 & $0.032 *$ \\
\hline Convexity $(\mathrm{mm})$ & $5.409 \pm 1.985$ & $4.636 \pm 2.146$ & -0.773 & 0.262 \\
\hline $\operatorname{SN}-P P\left(0^{\circ}\right)$ & $10.045 \pm 3.275$ & $9.545 \pm 3.602$ & 0.500 & 0.093 \\
\hline PP-MP $\left(^{\circ}\right)$ & $34.000 \pm 4.764$ & $34.727 \pm 4.390$ & 0.727 & 0.091 \\
\hline Occ-PP $\left(^{\circ}\right)$ & $20.500 \pm 4.806$ & $19.500 \pm 4.935$ & -1.000 & 0.139 \\
\hline Occ-MP $\left(^{\circ}\right)$ & $13.500 \pm 2.898$ & $15.318 \pm 2.648$ & 1.818 & $0.041^{*}$ \\
\hline U1-SN $\left(^{\circ}\right)$ & $98.591 \pm 6.587$ & $94.727 \pm 5.106$ & -3.864 & $0.028^{*}$ \\
\hline U-NA $\left(^{\circ}\right)$ & $21.045 \pm 3.971$ & $17.955 \pm 4.497$ & -3.091 & $0.033^{*}$ \\
\hline $\mathrm{U} 1-\mathrm{NA}(\mathrm{mm})$ & $2.727 \pm 1.348$ & $1.591 \pm 1.562$ & -1.136 & $0.017^{*}$ \\
\hline U1-FH (mm) (dental height) & $46.818 \pm 3.364$ & $50.045 \pm 3.876$ & 3.227 & $0.003^{* *}$ \\
\hline U1-PP (mm) (dental height) & $26.818 \pm 1.793$ & $28.682 \pm 1.722$ & 1.864 & $0.003^{* *}$ \\
\hline U6-FH (mm) (dental height) & $42.045 \pm 2.945$ & $43.591 \pm 3.590$ & 1.545 & $0.011 * *$ \\
\hline U6-PP (mm) (dental height) & $20.955 \pm 2.173$ & $21.500 \pm 2.439$ & 0.545 & $0.041^{*}$ \\
\hline \multicolumn{5}{|c|}{ Mandibular skeletal and dental } \\
\hline SNB $\left({ }^{\circ}\right)$ & $71.909 \pm 4.493$ & $72.273 \pm 4.297$ & 0.364 & 0.109 \\
\hline Facial angle $\left({ }^{\circ}\right)$ & $84.045 \pm 3.602$ & $84.045 \pm 3.395$ & 0.000 & 0.079 \\
\hline N. perpendicular-Pog (mm) & $11.139 \pm 5.595$ & $11.364 \pm 6.265$ & 0.227 & 0.646 \\
\hline Mandibular lenth(mm)(Cd-Gn) & $102.364 \pm 4.348$ & $105.545 \pm 4.942$ & 3.182 & $0.005^{* *}$ \\
\hline Ramus height $(\mathrm{mm})$ & $50.864 \pm 3.661$ & $52.409 \pm 3.056$ & 1.545 & $0.018^{*}$ \\
\hline Gonial angle $\left(^{\circ}\right)$ & $127.273 \pm 4.239$ & $127.364 \pm 4.267$ & 0.091 & 0.657 \\
\hline L1-MP $\left({ }^{\circ}\right)$ & $91.773 \pm 5.183$ & $92.091 \pm 4.505$ & 0.318 & 0.767 \\
\hline L1-NB $\left({ }^{\circ}\right)$ & $26.682 \pm 3.723$ & $27.636 \pm 4.154$ & 0.955 & 0.508 \\
\hline L1-NB (mm) & $4.727 \pm 1.403$ & $5.409 \pm 1.338$ & 0.682 & 0.091 \\
\hline L1-MP (mm) (dental height) & $38.000 \pm 2.280$ & $39.682 \pm 2.542$ & 1.682 & $0.003^{* *}$ \\
\hline L6-MP (mm) (dental height) & $31.182 \pm 1.820$ & $31.500 \pm 1.987$ & 0.318 & 0.308 \\
\hline \multicolumn{5}{|l|}{ Vertical } \\
\hline Facial axis $\left(^{\circ}\right)$ & $79.045 \pm 4.156$ & $79.773 \pm 3.573$ & 0.727 & 0.097 \\
\hline FMA $\left({ }^{\circ}\right)$ & $31.636 \pm 5.745$ & $32.682 \pm 4.771$ & 1.045 & 0.053 \\
\hline Facial height(\%)(UFH/LFH) & $0.751 \pm 0.049$ & $0.765 \pm 0.059$ & 0.014 & $0.028^{*}$ \\
\hline Lower face height $\left({ }^{\circ}\right)$ & $49.045 \pm 3.984$ & $48.227 \pm 3.228$ & -0.818 & 0.080 \\
\hline Posterior face height $(\mathrm{mm})(\mathrm{S}-\mathrm{Go})$ & $68.045 \pm 4.569$ & $69.500 \pm 5.153$ & 1.455 & 0.051 \\
\hline Anterior face height $(\mathrm{mm})(\mathrm{N}-\mathrm{Me})$ & $115.500 \pm 6.961$ & $118.182 \pm 7.561$ & 2.682 & $0.003 * *$ \\
\hline Jarabak ratio (\%) & $58.900 \pm 4.400$ & $58.600 \pm 3.900$ & -0.300 & 0.944 \\
\hline Mandibular arc $\left(^{\circ}\right)$ & $34.955 \pm 3.236$ & $34.682 \pm 3.282$ & -0.273 & 0.906 \\
\hline Anterior open-bite (mm) & $2.636 \pm 1.675$ & $0.000 \pm 1.265$ & 2.636 & $0.003^{* *}$ \\
\hline
\end{tabular}

were observed in the FR4 group (3.182 mm $\mathrm{p}<0.01,1.545 \mathrm{~mm} \mathrm{p}<0.05)$, these changes were considered to be non-significant when compared with the control.

Despite a higher incidence of vertical eruption of the lower incisors, comparison of the two groups showed no significant difference $(p>0.05)$.

Vertical Changes (Tables III, IV, V)

The decrease in the amount of open-bite $(\mathrm{mm})$ was found to be crucial when the groups were compared.

In the treatment and control groups the degree of open-bite was decreased by 
Table IV

Longitudinal changes in the control group

\begin{tabular}{|c|c|c|c|c|}
\hline Parameter & $\begin{array}{c}\text { Preobservation } \\
(\bar{x} \pm S D)\end{array}$ & $\begin{array}{c}\text { Postobservation } \\
(\bar{x} \pm S D)\end{array}$ & Difference & $\mathrm{p}$ value \\
\hline \multicolumn{5}{|l|}{ Maxillary skeletal and dental } \\
\hline SNA $\left(^{\circ}\right)$ & $78.450 \pm 7.065$ & $78.950 \pm 6.322$ & 0.500 & 0.445 \\
\hline N. perpendicular-A (mm) & $-1.800 \pm 3.450$ & $-1.150 \pm 3.473$ & 0.650 & 0.059 \\
\hline Maxillary length(mm) (Cd-A) & $75.150 \pm 4.710$ & $77.400 \pm 4.569$ & 2.250 & $0.005^{* *}$ \\
\hline Convexity $(\mathrm{mm})$ & $3.700 \pm 1.874$ & $3.800 \pm 1.719$ & 0.100 & 0.584 \\
\hline $\mathrm{SN}-\mathrm{PP}\left({ }^{\circ}\right)$ & $8.900 \pm 4.683$ & $8.350 \pm 4.755$ & -0.550 & 0.173 \\
\hline PP-MP $\left({ }^{\circ}\right)$ & $32.600 \pm 4.142$ & $32.350 \pm 4.217$ & -0.250 & 0.499 \\
\hline Occ-PP $\left({ }^{\circ}\right)$ & $17.405 \pm 3.715$ & $16.550 \pm 2.544$ & -0.900 & 0.554 \\
\hline Occ-MP $\left({ }^{\circ}\right)$ & $15.150 \pm 2.161$ & $15.800 \pm 4.780$ & 0.650 & 0.859 \\
\hline $\mathrm{U} 1-\mathrm{SN}\left({ }^{\circ}\right)$ & $104.050 \pm 5.718$ & $103.950 \pm 5.408$ & -0.100 & 0.722 \\
\hline $\mathrm{U} 1-\mathrm{NA}\left({ }^{\circ}\right)$ & $25.900 \pm 6.887$ & $25.100 \pm 4.533$ & -0.800 & 0.554 \\
\hline $\mathrm{U} 1-\mathrm{NA}(\mathrm{mm})$ & $2.850 \pm 1.415$ & $2.850 \pm 1.765$ & 0.000 & 0.636 \\
\hline U1-FH (mm) (dental height) & $43.150 \pm 4.177$ & $44.900 \pm 3.307$ & 1.750 & $0.009 * *$ \\
\hline U1-PP (mm) (dental height) & $24.100 \pm 3.035$ & $25.450 \pm 2.629$ & 1.350 & $0.012^{*}$ \\
\hline U6-FH (mm) (dental height) & $34.450 \pm 2.477$ & $39.800 \pm 2.275$ & 5.350 & $0.015^{*}$ \\
\hline U6-PP (mm) (dental height) & $19.400 \pm 1.524$ & $20.000 \pm 1.826$ & 0.600 & 0.091 \\
\hline \multicolumn{5}{|l|}{ Mandibular skeletal and dental } \\
\hline $\operatorname{SNB}\left({ }^{\circ}\right)$ & $74.350 \pm 6.223$ & $74.850 \pm 5.845$ & 0.500 & 0.236 \\
\hline Facial angle $\left({ }^{\circ}\right)$ & $83.700 \pm 2.965$ & $84.550 \pm 3.113$ & 0.850 & $0.017^{*}$ \\
\hline N. perpendicular-Pog (mm) & $11.000 \pm 5.533$ & $10.100 \pm 4.971$ & -0.900 & 0.263 \\
\hline Mandibular length(mm)(Cd-Gn) & $98.750 \pm 5.633$ & $101.900 \pm 5.607$ & 3.150 & $0.008 * *$ \\
\hline Ramus height (mm) & $48.000 \pm 5.121$ & $49.200 \pm 4.803$ & 1.200 & $0.012^{*}$ \\
\hline Gonial angle $\left(^{\circ}\right)$ & $127.400 \pm 4.458$ & $126.200 \pm 4.104$ & -1.200 & $0.041^{*}$ \\
\hline L1-MP $\left({ }^{\circ}\right)$ & $92.900 \pm 5.806$ & $92.900 \pm 6.471$ & 0.000 & 0.953 \\
\hline L1-NB $\left({ }^{\circ}\right)$ & $28.100 \pm 5.705$ & $27.800 \pm 5.841$ & -0.300 & 0.813 \\
\hline L1-NB (mm) & $5.150 \pm 2.334$ & $5.500 \pm 2.369$ & 0.350 & 0.080 \\
\hline L1-MP (mm) (dental height) & $36.500 \pm 2.582$ & $37.300 \pm 2.830$ & 0.800 & $0.012^{*}$ \\
\hline L6-MP (mm) (dental height) & $29.750 \pm 2.276$ & $29.750 \pm 2.276$ & 0.000 & 1.000 \\
\hline \multicolumn{5}{|l|}{ Vertical } \\
\hline Facial axis $\left({ }^{\circ}\right)$ & $80.950 \pm 3.166$ & $81.550 \pm 3.387$ & 0.600 & 0.401 \\
\hline FMA $\left(^{\circ}\right)$ & $32.150 \pm 3.266$ & $31.450 \pm 3.640$ & -0.700 & 0.107 \\
\hline Facial height(\%)(UFH/LFH) & $0.766 \pm 0.081$ & $0.773 \pm 0.057$ & 0.007 & 0.590 \\
\hline Lower face height $\left({ }^{\circ}\right)$ & $48.400 \pm 3.017$ & $48.100 \pm 3.026$ & -0.300 & 0.674 \\
\hline Posterior face height(mm)(S-Go) & $66.850 \pm 5.011$ & $68.550 \pm 4.884$ & 1.700 & $0.008^{* *}$ \\
\hline Anterior face height $(\mathrm{mm})(\mathrm{N}-\mathrm{Me})$ & $111.100 \pm 3.872$ & $112.600 \pm 4.427$ & 1.500 & $0.008^{* *}$ \\
\hline Jarabak ratio $(\%)$ & $59.900 \pm 4.500$ & $60.600 \pm 3.900$ & 0.700 & 0.128 \\
\hline Mandibular $\operatorname{arc}\left({ }^{\circ}\right)$ & $33.250 \pm 4.316$ & $33.200 \pm 3.584$ & -0.050 & 0.889 \\
\hline Anterior open-bite (mm) & $3.800 \pm 1.317$ & $2.700 \pm 1.567$ & 1.100 & $0.024 *$ \\
\hline
\end{tabular}

${ }^{*} \mathrm{p}<0.05$

$* * \mathrm{p}<0.01$

$2.636 \mathrm{~mm}(\mathrm{p}<0.01)$ and $1.100 \mathrm{~mm}(\mathrm{p}<0.05)$, respectively, and the difference between the groups was found to be significant $(\mathrm{p}<0.01)$.

Anterior face height increased to $2.682 \mathrm{~mm}(\mathrm{p}<0.01)$, which was significant in comparison with the control $(\mathrm{p}<0.05)$. 
Table V

Comparison of treatment changes between FR4 and control groups

\begin{tabular}{|c|c|c|c|c|}
\hline Parameter & $\begin{array}{c}\text { Experimental } \\
(\bar{x} \pm S D)\end{array}$ & $\begin{array}{l}\text { Control } \\
(\bar{x} \pm S D)\end{array}$ & $\mathrm{U}$ & $\mathrm{p}$ value \\
\hline \multicolumn{5}{|l|}{ Maxillary skeletal and dental } \\
\hline SNA $\left(^{\circ}\right)$ & $-0.455 \pm 0.986$ & $0.500 \pm 1.650$ & 36 & 0.165 \\
\hline N. perpendicular-A (mm) & $-0.818 \pm 1.210$ & $0.650 \pm 0.973$ & 15 & $0.004 * *$ \\
\hline Maxillary length(mm) (Cd-A) & $1.227 \pm 1.489$ & $2.250 \pm 1.379$ & 33 & 0.119 \\
\hline Convexity $(\mathrm{mm})$ & $-0.773 \pm 1.679$ & $0.100 \pm 1.022$ & 38 & 0.207 \\
\hline SN-PP $\left({ }^{\circ}\right)$ & $-0.500 \pm 0.894$ & $-0.550 \pm 1.117$ & 54 & 0.943 \\
\hline PP-MP $\left(^{\circ}\right)$ & $0.727 \pm 1.191$ & $-0.250 \pm 1.137$ & 81 & 0.061 \\
\hline Occ-PP $\left({ }^{\circ}\right)$ & $-1.000 \pm 2.408$ & $-0.900 \pm 3.703$ & 48 & 0.621 \\
\hline Occ-MP $\left({ }^{\circ}\right)$ & $1.818 \pm 2.272$ & $0.650 \pm 4.110$ & 73 & 0.203 \\
\hline $\mathrm{U} 1-\mathrm{SN}\left({ }^{\circ}\right)$ & $-3.864 \pm 4.050$ & $-0.100 \pm 3.502$ & 29 & 0.061 \\
\hline $\mathrm{U} 1-\mathrm{NA}\left({ }^{\circ}\right)$ & $-3.091 \pm 3.974$ & $-0.800 \pm 4.917$ & 41 & 0.329 \\
\hline $\mathrm{U} 1-\mathrm{NA}(\mathrm{mm})$ & $-1.136 \pm 1.142$ & $0.000 \pm 1.434$ & 26 & $0.035^{*}$ \\
\hline Ul-FH (mm) (dental height) & $3.227 \pm 1.708$ & $1.750 \pm 1.318$ & 82 & 0.060 \\
\hline U1-PP (mm) (dental height) & $1.864 \pm 1.185$ & $1.350 \pm 1.156$ & 69 & 0.318 \\
\hline U6-FH (mm) (dental height) & $1.545 \pm 1.368$ & $5.350 \pm 1.248$ & 61 & 0.696 \\
\hline U6-PP (mm) (dental height) & $0.545 \pm 0.611$ & $0.600 \pm 0.937$ & 58 & 0.827 \\
\hline \multicolumn{5}{|l|}{ Mandibular skeletal and dental } \\
\hline SNB $\left(^{\circ}\right)$ & $0.364 \pm 0.778$ & $0.500 \pm 1.247$ & 41 & 0.285 \\
\hline Facial angle $\left({ }^{\circ}\right)$ & $0.000 \pm 1.871$ & $0.850 \pm 0.784$ & 35 & 0.155 \\
\hline N. perpendicular-Pog (mm) & $0.227 \pm 2.805$ & $-0.900 \pm 2.221$ & 72 & 0.229 \\
\hline Mandibular length(mm)(Cd-Gn) & $3.182 \pm 2.160$ & $3.150 \pm 1.796$ & 57 & 0.915 \\
\hline Ramus height (mm) & $1.545 \pm 1.457$ & $1.200 \pm 0.789$ & 62 & 0.613 \\
\hline Gonial angle $\left({ }^{\circ}\right)$ & $0.091 \pm 1.841$ & $-1.200 \pm 1.585$ & 80 & 0.081 \\
\hline L1-MP $\left(^{\circ}\right)$ & $0.318 \pm 3.002$ & $0.000 \pm 2.877$ & 61 & 0.671 \\
\hline L1-NB ( $\left(^{\circ}\right)$ & $0.955 \pm 3.402$ & $-0.300 \pm 2.898$ & 63 & 0.571 \\
\hline L1-NB (mm) & $0.682 \pm 1.290$ & $0.350 \pm 0.580$ & 62 & 0.603 \\
\hline L1-MP (mm) (dental height) & $1.682 \pm 1.210$ & $0.800 \pm 0.587$ & 81 & 0.062 \\
\hline L6-MP (mm) (dental height) & $0.318 \pm 0.956$ & $0.000 \pm 0.527$ & 72 & 0.219 \\
\hline \multicolumn{5}{|l|}{ Vertical } \\
\hline Facial axis $\left({ }^{\circ}\right)$ & $0.727 \pm 1.367$ & $0.600 \pm 1.792$ & 62 & 0.644 \\
\hline FMA $\left(^{\circ}\right)$ & $1.045 \pm 1.739$ & $-0.700 \pm 1.229$ & 87 & $0.023^{*}$ \\
\hline Facial height(\%)(UFH/LFH) & $0.014 \pm 0.018$ & $0.007 \pm 0.034$ & 67 & 0.369 \\
\hline Lower face height $\left(^{\circ}\right)$ & $-0.818 \pm 1.505$ & $-0.300 \pm 1.874$ & 47 & 0.569 \\
\hline Posterior face height $(\mathrm{mm})(\mathrm{S}-\mathrm{Go})$ & $1.455 \pm 2.006$ & $1.700 \pm 1.438$ & 56 & 0.972 \\
\hline Anterior face height $(\mathrm{mm})(\mathrm{N}-\mathrm{Me})$ & $2.682 \pm 1.488$ & $1.500 \pm 0.850$ & 83 & $0.042^{*}$ \\
\hline Jarabak ratio $(\%)$ & $-0.300 \pm 0.015$ & $0.700 \pm 0.013$ & 38 & 0.204 \\
\hline Mandibular arc $\left(^{\circ}\right)$ & $-0.273 \pm 2.630$ & $-0.050 \pm 2.409$ & 56 & 0.972 \\
\hline Anterior open-bite (mm) & $2.636 \pm 0.710$ & $1.100 \pm 1.101$ & 98 & $0.002 * *$ \\
\hline
\end{tabular}

${ }^{*} \mathrm{p}<0.05$

** $\mathrm{p}<0.01$

\section{Discussion}

Although the treatment period used here of one year and two months was less than the proposed Fränkel treatment duration, the changes observed during this period gave sufficient information about the efficiency of FR4.

There has been only one study on the effects of the FR4 appliance since that of FRÄNKEL AND FRÄNKEL ${ }^{[18]}$. OWEN ${ }^{[21]}$ reported the results of treatment of some open-bite patients using the FR4. Accordingly, we shall compare our results with those of FRÄNKEL AND FRÄNKEL ${ }^{[18,19]}$. 
The FR4 appliance was found to affect the changes in dental structures rather than skeletal configuration.

Although not significant when compared with the control, vertical eruption of the upper and lower incisors in the FR4 group was found more frequently. This vertical eruption of incisors in the FR4 group combined with the retraction of the upper incisors which would have affected the vertical height, were considered to be the main reasons for the decrease in open-bite. This change may have resulted from the lip seal exercises and the change from mouth breathing to nasal breathing, which in turn would have caused the tongue to alter its postural position backward, thus allowing the incisors to erupt freely.

Our findings show that the use of the FR4 appliance caused some backward rotation of the mandible (FMA: $1.045^{\circ}$ ), which was significant when compared with the control. However, this contradicts the findings of Fränkel's study, where appliance caused anterior rotation of the mandible, whereas backward rotation of the mandible continued in his control sample.

In addition, the fact that the increase in the anterior face height in our experimental group was significantly greater than in the control suggests that the appliance restricts the natural anterior rotation of the mandible, as seen in the control sample.

FRÄNKEL AND FRÄNKEL ${ }^{[18,19]}$ explained the forward rotation of the mandible as an increase in posterior face height, which they attributed to compensatory growth at the condyle and raising of the anterior part of the mandible as a result of lip seal exercises. No such anterior rotation of the mandible was observed in our group, although they performed lip seal exercises throughout the treatment, and no significant increase in ramus height was observed in compaison with the controls.

FRÄNKEL AND FRÄNKEL ${ }^{[18,19]}$ stated that in their experimental group, posterior maxillary and mandibular dentoalveolar growth was not inhibited by use of the appliance, although they did not believe that maxillary dentoalveolar excess was a factor causing open-bite. We also found that the use of FR4 did not change the normal eruption of the upper and lower first molars in compaison with the control. FRäNKEL AND FRÄNKEL ${ }^{[18,19]}$ set out from Nahoum's finding that the distance from the maxillary first molar to the palatal plane was not significantly different from that in normal subjects. More recent research has shown that posterior maxillary dentoalveolar excess is a significant finding in open-bite cases. From this viewpoint, this is one area that has to be controlled during the treatment of skeletal open-bite.

Importance of vertical control in the treatment of malocclusion has been stressed many times ${ }^{[1,8,15,21]}$. OWEN ${ }^{[21]}$ stated that the FR4 appliance did not prove effective in his study, in agreement with our results. He modified the appliance by adding a posterior bite block and tubes for occipital-pull head-gear for positive control of the posterior maxilla.

McN $_{\text {AMARA }}{ }^{[22]}$ stated that patients with an excessive vertical dimension were least likely to benefit from the Fränkel treatment, and therefore he combined the FR4 appliance with a vertical-pull chin cap in patients with skeletal open-bite.

It is not clear why excessive eruption of posterior theeth, causing backward 
rotation of the mandible, occurs in children with open-bite, although the occlusal forces are not low during this period in comparison with normal individuals. The findings of PROFFIT's group ${ }^{[23,24]}$ suggest that the long face pattern present in children when occlusal forces are not low, is not a cause of, but rather an effect of this condition. INGERVALL et al. ${ }^{[25]}$ in their study concluded that the long face morphology characterisitc of mouth-breathing children, is not due to weak muscles.

The theory of soft tissue stretching proposed by SoLow et al. ${ }^{[26]}$ states that in upper airway inadequacy a mouth breather will alter his head posture, and that this in turn will affect craniofacial morphology. This change in head posture may increase the interocclusal space, causing excessive eruption of posterior teeth.

Considering the results of these studies, it seems improbable that lip seal exercises, which are highly recommended by FRÄNKEL AND FRÄNKEL ${ }^{[18]}$ can alter growth direction by strengthening the elevator muscles, which in any case are not weak during this period.

Further research on this subject may result in different conclusions, and by focusing treatment planning on the cause of the vertical excess, it should be possible to alter the direction of growth in the early mixed dentition period.

\section{Summary and Conclusions}

The effects of the FR4 appliance in cases of skeletal open-bite were evaluated cephalometrically and the following conclusions reached:

1-The FR4 appliance did not produce any skeletal changes.

2-No significant changes in facial proportions occurred.

3-The lack of any significant increase in ramus height and an unexpected slight posterior rotation of the mandible contradict the hypothesis on which this appliance is based.

4-The amount of open-bite decreased significantly in the FR4 group. Vertical eruption of the upper and lower incisors and retraction of the upper incisors are considered responsible for the closure of open-bite.

The FR4 appliance was found to affect dental structures rather than skeletal configuration, thus failing to improve the facial pattern, and merely masking the existing vertical problem.

\section{References}

[1] Nahoum, H. I.: Vertical proportions and the palatal plane in anterior open-bite, Am. J. Orthodont., 59, 273-282, 1971

[2] Lopez-Gavito, G., Wallen, T. R., Little, R. M. and Joondeph, D. R.: Anteroir open-bite malocclusion: a longitudinal 10-year postretention evaluation of orthodontically treated patients, Am. J. Orthodont., 87, 175-186, 1985

[3] Cangialosi, T. J.: Skeletal morphologic features of anterior open-bite, Am. J. Orthodont., 85, 28-36, 1984

[4] Nahoum, H. I.: Anterior open-bite; a cephalometric analysis and suggested treatment procedures, Am. J. Orthodont., 67, 513-520, 1975

[5] Nahoum, H. I. and Horowitz, S. L.: Varieties of anterior open-bite, Am. J. Orthodont., 61, 486-492, 1972

[6] Ellis, E. and McNamara, J. A. Jr.: Components of adult class III open-bite malocclusion, 
Am. J. Orthodont., 86, 277-290, 1984

[7] Jones, O. G.: A cephalometric study of 32 North American black patients with anterior open-bite, Am. J. Orthodont. Dentofac. Orthoped., 95, 289-296, 1989

[8] Schendel, S. A., Eisenfeld, J., Bell, W. H., Epker, B. N. and Mishelevich, D. J.: The long face syndrome; vertical maxillary excess, Am. J. Orthodont., 70, 398-408, 1976

[9] Epker, B. N. and Fish, L. C.: Surgical-orthodontic correction of open-bite deformity, Am. J. Orthodont., 71, 278-299, 1977

[10] Proffit, W. R. and Bell, W. H.: Open bite. In Surgical Correction of Dentofacial Deformities, 1058-1209, Bell, W. H., Proffit, W. R., White, R. P., eds., W. B. Saunders, Philadelphia, U.S.A., 1980

[11] Van der Linden, F.P.M.G. and Boersma, H.: Etiology. In Diagnosis and Treatment Planning in Dentofacial Orthopedics, 17-37, VAN DER Linden, F.P.M.G., ed., Quintessence Publishing Co., London, U.K., 1987

[12] Graber, T. M. and Swain, B. F.: Dentofacial Orthopedics. In Current Orthodontic Concepts and Techniques, Vol II, 365-452, Graber, T. M., ed., W. B. Saunders, Philadelphia, U.S.A., 1975

[13] Woodside, D. G. and Linder-Aranson, S.: Progressive increase in lower anterior face height and the use of posterior occlusal bite-block in its management. In: Orthodontics. State of the Art: Essence of the Science, 200-221, Graber, L. W., ed., C.V. Mosby, St. Louis, U.S.A., 1989

[14] Dellinger, E. L.: Orthodontic correction of the long-face syndrome, Ear Nose Throat J., 66, 237-241, 1987

[15] KIM, Y. H.: Anterior open-bite and its treatment with multiloop edgewise archwire, Angle Orthodont., 57, 290-321, 1987

[16] Huang, G., Justus, R., Kennedy, D. B. and Kokich, V. G.: Stability of anterior open-bite treated with crib therapy, Angle Orthodont., 60, 17-24, 1990

[17] Moss, M. L. and Salentijn, L.: Differences between the functional matrices in anterior open-bite and in deep overbite, Am. J. Orthodont., 60, 264-280, 1971

[18] FrÄNKEL, R. and FräNKEL, C.: A functional approach to treatment of skeletal open-bite, Am. J. Orthodont., 84, 54-68, 1983

[19] FrÄNKEL, R. and FräNKEL, C.: The function regulator in the treatment of skeletal open-bite. In Orofacial Orthopedics with the Function Regulator, 73-89, Karger, Basel, 1989

[20] Graber, T. M., Rakosi, T. and Petrovic, A. G.: The Fränkel Function Regulator. In Dentofacial Orthopedics with Functional Appliances, 219-274, Graber, T. M. ed., C.V. Mosby, St. Louis, U.S.A., 1985

[21] Owen, A. H.: Modified function regulator for vertical maxillary excess, J. Clin. Orthodont., 19, 733-749, 1985

[22] McNamara, J. A. Jr.: On the Fränkel appliance, part-2, Clinical Management, J. Clin. Orthodont., 16, 390-407, 1982

[23] Proffit, W. R., Fields, H. W. and Nixon, W. L.: Occlusal forces in normal and long-face adults, J. Dent. Res., 62, 566-570, 1983

[24] Proffit, W. R. and Fields, H. W.: Occlusal forces in normal and long-face children, J. Dent. Res., 62, 571-574, 1983

[25] Ingervall, B., Thuer, V. and Kuster, R.: Lack of correlation between mouth-breathing and bite force, Eur. J. Orthodont., 11, 43-46, 1989

[26] Solow, B., Siersbaek-Nielsen, S. and Greve, E.: Airway adequacy, head posture and craniofacial morphology, Am. J. Orthodont., 86, 214-223, 1984

[27] FränKEL, R.: Lip seal training in treatment of open-bite, Eur. J. Orthodont., 62, 113-141, 1972 